\title{
Article \\ Optimized Growth and Laser Application of Yb:LuAG Single-Crystal Fibers by Micro-Pulling-Down Technique
}

\author{
Anye Wang, Jian Zhang *D, Shuai Ye, Xiaofei Ma, Baiyi Wu, Siyuan Wang, Feifei Wang, Tao Wang, Baitao Zhang \\ and Zhitai Jia (D)
}

check for

updates

Citation: Wang, A.; Zhang, J.; Ye, S.; Ma, X.; Wu, B.; Wang, S.; Wang, F.; Wang, T.; Zhang, B.; Jia, Z. Optimized Growth and Laser Application of $\mathrm{Yb}$ :LuAG Single-Crystal Fibers by Micro-Pulling-Down Technique. Crystals 2021, 11, 78. https:// doi.org/10.3390/cryst11020078

Received: 5 January 2021

Accepted: 18 January 2021

Published: 20 January 2021

Publisher's Note: MDPI stays neutral with regard to jurisdictional claims in published maps and institutional affiliations.

Copyright: (c) 2021 by the authors. Licensee MDPI, Basel, Switzerland. This article is an open access article distributed under the terms and conditions of the Creative Commons Attribution (CC BY) license (https:/ / creativecommons.org/licenses/by/ $4.0 /)$.
State Key Laboratory of Crystal Materials, Shandong University, Jinan 250100, China; way5268@163.com (A.W.); sye1120@163.com (S.Y.); 17853143775@163.com (X.M.); 18769783256@163.com (B.W.);

15064100373@163.com (S.W.); ffwang0@163.com (F.W.); crystalwtsdu@163.com (T.W.); btzhang@sdu.edu.cn (B.Z.); z.jia@sdu.edu.cn (Z.J.)

* Correspondence: jian.zhang@sdu.edu.cn

\begin{abstract}
Single-crystal fibers (SCFs) have a great application potential in high-power lasers due to their excellent performance. In this work, high-quality and crack-free $\mathrm{Yb}^{3+}: \mathrm{Lu}_{3} \mathrm{Al}_{5} \mathrm{O}_{12}(\mathrm{Yb}: \mathrm{LuAG})$ SCFs were successfully fabricated by the micro-pulling-down ( $\mu$-PD) technology. Based on the laser micrometer and the X-ray Laue diffraction results, these Yb:LuAG SCFs have a less than 5\% diameter fluctuation and good crystallinity along the axial direction. More importantly, the distribution of $\mathrm{Yb}$ ions is proved to be uniform by electron probe microanalysis (EPMA) and the scanning electron microscope (SEM). In the laser experiment, the continuous-wave (CW) output power using a $1 \mathrm{~mm}$ diameter $\mathrm{Yb}: \mathrm{LuAG}$ single-crystal fiber is determined to be $1.96 \mathrm{~W}$, at the central wavelength of $1047 \mathrm{~nm}$, corresponding to a slope efficiency of $13.55 \%$. Meanwhile, by applying a $3 \mathrm{~mm}$ diameter Yb:LuAG SCF, we obtain a $4.7 \mathrm{~W}$ CW laser output at $1049 \mathrm{~nm}$ with the slope efficiency of $22.17 \%$. The beam quality factor $\mathrm{M}^{2}$ is less than 1.1 in both conditions, indicating a good optical quality of the grown fiber. Our results show that the $\mathrm{Yb}$ :LuAG SCF is a potential solid-state laser gain medium for $1 \mu \mathrm{m}$ high-power lasers.
\end{abstract}

Keywords: single-crystal fiber; micro-pulling-down; Yb:LuAG; CW laser

\section{Introduction}

In recent years, as the most widely investigated laser system, solid-state lasers have become a research hotspot due to their high laser gain coefficient, high photoelectric conversion efficiency, stable working medium and compact laser system [1-4]. The laser gain medium has a pronounced influence on the performance of the lasers. Subject to the nature and structure of the traditional solid-state gain medium, mainly including bulk crystals, glass and ceramics, a further increase in the laser output power is limited. In spite of that, the invention and manufacture of a glass fiber laser, which is considered as the "third-generation laser", have brought new development ideas for solid-state lasers. Due to the ideal beam quality, high conversion efficiency, being maintenance-free, high stability and small size, a single glass fiber laser can achieve a $10 \mathrm{~kW} \mathrm{CW} \mathrm{laser} \mathrm{output.} \mathrm{However,}$ due to the low thermal conductivity and lower mechanical strength, the output power of glass fiber lasers is also limited. Therefore, it is necessary to find a new type of laser gain medium [5,6].

Single-crystal fibers (SCFs) are a kind of one-dimensional laser gain medium between traditional bulk crystals and glass fibers. They combine the advantages of bulk crystals and glass fibers, such as good physical properties and excellent thermal management performances. Therefore, they are expected to solve the current problems encountered by glass fiber lasers. Compared with silica fibers, SCFs have higher thermal conductivity, a higher rare-earth ion doping concentration, a lower nonlinear effect and a higher laser damage threshold. Their theoretical laser output power is more than 50 times that of 
traditional silica glass fibers [7]. In 2012, Xavier Delen et al. obtained a 251 W CW laser output using a $\mathrm{Yb}^{3+}: \mathrm{Y}_{3} \mathrm{Al}_{5} \mathrm{O}_{12}$ (Yb:YAG) SCF grown by $\mu$-PD technology [8].

As for the laser gain medium, rare-earth garnet crystals are good candidates because they have excellent physical and mechanical properties. They belong to the cubic crystal system, Ia3d space group, and representative crystals include $\mathrm{Y}_{3} \mathrm{Al}_{5} \mathrm{O}_{12}$ (YAG), $\mathrm{Lu}_{3} \mathrm{Al}_{5} \mathrm{O}_{12}$ (LuAG) and $\mathrm{Y}_{3} \mathrm{Sc}_{2} \mathrm{Ga}_{3} \mathrm{O}_{12}$ (YSGG). LuAG with a melting point of $2333 \mathrm{~K}$ and a density of $6.67 \mathrm{~g} / \mathrm{cm}^{3}$ is a kind of garnet structure crystal with excellent thermal and optical properties, a high laser damage threshold and high thermal conductivity. Compared with YAG, the thermal conductivity of LuAG crystals is less affected by the increasing concentration of doping ions, which is conducive to achieve the miniaturization and integration of lasers [9-15]. In addition, Yb:LuAG has a larger effective emission cross-sectional area than $\mathrm{Yb}: Y A G$, which is more suitable for high-power solid-state lasers [16].

$\mathrm{Yb}: \mathrm{LuAG}$ crystals are considered to be high-quality laser gain mediums for $1 \mu \mathrm{m}$ band lasers. The $\mathrm{Yb}^{3+}$ absorption band is located at $900 \sim 1000 \mathrm{~nm}$, which can be effectively coupled with an InGaAs laser diode without strictly controlling the temperature; compared with $\mathrm{Nd}^{3+}, \mathrm{Yb}^{3+}$ has a weaker concentration quenching effect, which is also beneficial to the miniaturization of lasers. Furthermore, its absorption wavelength $(900-980 \mu \mathrm{m})$ and emission wavelength (980-1100 $\mu \mathrm{m})$ reduce the thermal load effect, which leads to high quantum efficiency almost up to $90 \%$. Meanwhile, considering that $\mathrm{Yb}^{3+}$ laser crystals have only the ground state ${ }^{2} \mathrm{~F}_{7 / 2}$ and the excited state ${ }^{2} \mathrm{~F}_{5 / 2}$, and there are no other laser state energy levels above ${ }^{2} \mathrm{~F}_{5 / 2}$, parasitic effects such as up-conversion, excited-state absorption and relaxation oscillations can be avoided to reduce laser energy loss [17-19]. In recent years, some progress has been achieved in the research of 1 micron band lasers for $\mathrm{Yb}: \mathrm{LuAG}$ SCFs. In 2009, the University of Lyon achieved a continuous laser output of $3.3 \mathrm{~W}$ with $100 \mathrm{~W}$ pump power by using a $1 \mathrm{~mm}$ diameter $\mathrm{Yb}$ :LuAG fiber. In 2012, the University of Pisa reached a high efficiency of $32 \%$ with a $3 \mathrm{~mm}$ diameter Yb:LuAG SCF, but the output power was only $23 \mathrm{~mW}[20,21]$. Therefore, further research on laser application of $\mathrm{Yb}: \mathrm{LuAG}$ SCFs is of great significance.

At present, the main growth methods of SCFs include the laser-heated pedestal growth (LHPG) method and $\mu$-PD technology. The LHPG method has the advantages of high growth speed without crucibles. However, due to the large temperature gradient at the growth interface, the as-grown crystal fibers have large thermal stress, resulting in the decrease in optical uniformity. Compared with LHPG, our self-designed $\mu$-PD device improves the homogeneity of melt components and makes the shape of the fiber controllable. In addition, the temperature gradient during SCF growth can be reduced by using the after-heater and temperature field, so the crystals have a higher laser damage threshold [22-24]. In this work, we successfully grow high-quality SCFs using $\mu$-PD technology. The systematical characterizations on the obtained Yb:LuAG SCF prove its low diameter fluctuation, good crystallinity and uniformity of doped ions. The continuouswave laser performance of the $\mathrm{Yb}: \mathrm{LuAG}$ SCFs is also investigated.

\section{Materials and Methods}

\subsection{Growth of Single-Crystal Fibers}

The raw materials of $\mathrm{Yb}^{3+}$ :LuAG were synthesized by a solid-state reaction. $\mathrm{Yb}_{2} \mathrm{O}_{3}$, $\mathrm{Lu}_{2} \mathrm{O}_{3}$ and $\mathrm{Al}_{2} \mathrm{O}_{3}$ oxide powders of $4 \mathrm{~N}$ purity were weighed according to a stoichiometric ratio of $\mathrm{Yb}_{\mathrm{x}} \mathrm{Lu}_{(3-\mathrm{x})} \mathrm{Al}_{5} \mathrm{O}_{12}(\mathrm{x}=0.21,0.3)$. The powders were ground in an agate mortar for 20 30 $\mathrm{min}$ to ensure uniform mixing. Then, the powder materials were pressed into wafers under the pressure of $50 \mathrm{Mpa}$ and sintering was carried out in a muffle furnace at $1873 \mathrm{~K}$ for $24 \mathrm{~h}$.

High-quality $\mathrm{Yb}: \mathrm{LuAG}$ single-crystal fibers were grown by the self-developed $\mu$-PD growth equipment. The schematic diagram is shown in Figure 1. About $3 \sim 5 \mathrm{~g}$ sintered polycrystalline materials was put into an iridium crucible and melted by radio frequency (RF) induction heating (power: $\sim 3850 \mathrm{~W}$ ). The melt remained stable in the capillary at the bottom of the crucible under the force of gravity and surface tension. A high-quality LuAG 
crystal $\left(1 \times 1 \times 30 \mathrm{~mm}^{3}\right)$ in the $<111>$ direction was used as seed. The seed crystal rose to the bottom of the crucible to contact the melt. After the melt fully contacted the seed crystal and the melting zone was stable, the seed crystal started to be pulled downward and the single-crystal fiber started to grow. In order to avoid oxidation of the iridium crucible, we vacuumed the furnace chamber to below $30 \mathrm{~Pa}$ and then filled it with dry argon to atmospheric pressure for crystal growth. The different stages of the Yb:LuAG SCFs' growth process could be observed in real time using a CCD camera through the observation window (Figure 2).

(a)

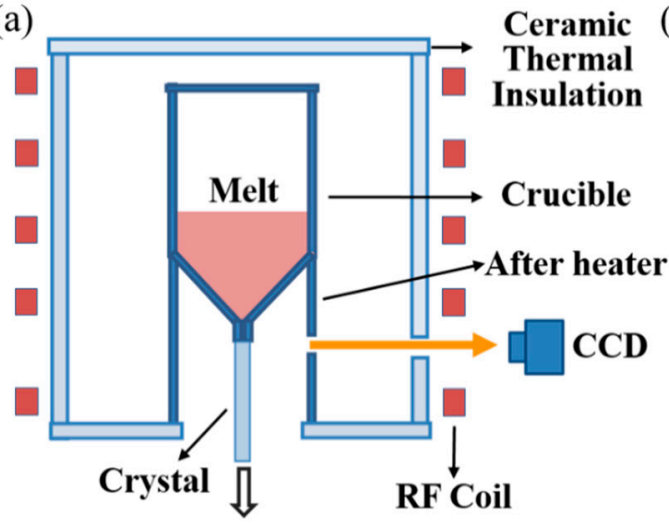

(b)

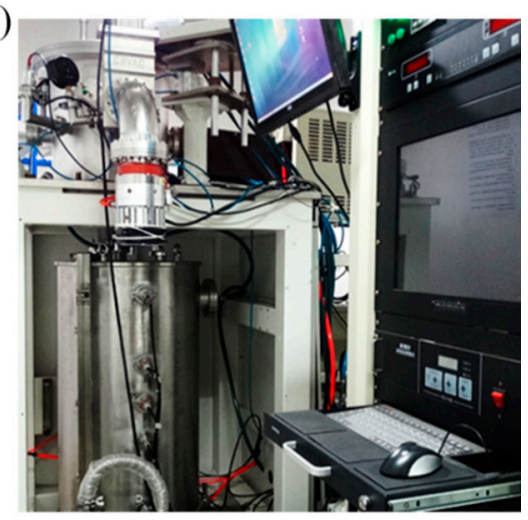

Figure 1. Schematic diagram (a) and equipment photo $(\mathbf{b})$ of the micro-pulling-down $(\mu-\mathrm{PD})$ technique.

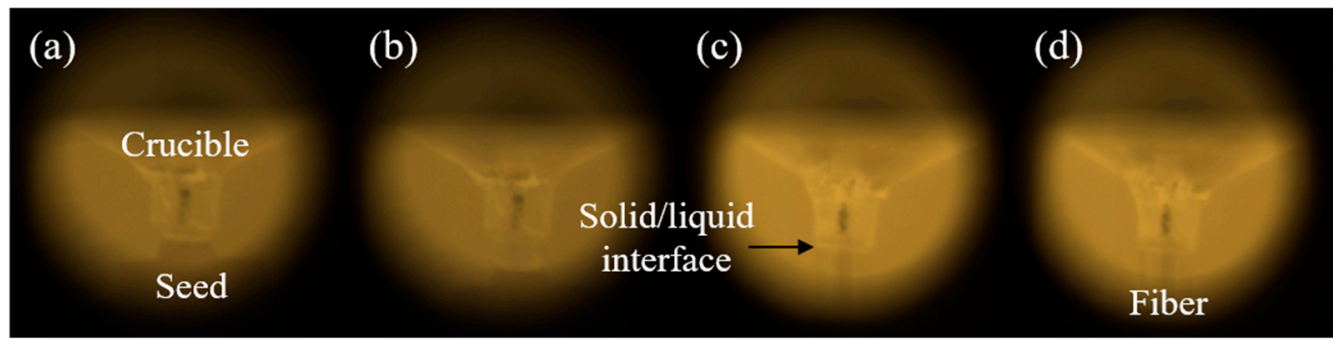

Figure 2. Real-time images of seeding $(\mathbf{a}, \mathbf{b})$ and stable crystal growth $(\mathbf{c}, \mathbf{d})$ by the CCD camera.

The iridium after-heater with a small size ( $\Phi 2$ and $\Phi 4 \mathrm{~mm})$ of the observation window and two layers of ceramic thermal insulation were used to reduce the temperature gradient during crystal growth. Crucibles with 1 and $3 \mathrm{~mm}$ nozzles were used for crystal growth. A slow crystal growth rate is conducive to improving the optical quality and crystallinity. In this way, the speed used in this work was $1 \sim 3 \mathrm{~mm} / \mathrm{h}$. When the $3 \mathrm{~mm}$ diameter fiber is grown, the growth rate of $>3 \mathrm{~mm} / \mathrm{h}$ will reduce the crystallinity. According to our experience, the growth rate of $1 \mathrm{~mm} / \mathrm{h}$ is enough to obtain high-quality crystals. During the growth process, it is necessary to increase the powers according to the crystal growth speed and fiber diameter, as to balance heat loss.

An excellent Yb:LuAG SCF was grown successfully by the $\mu$-PD technique. As it is exhibited in Figure 3, the length of the obtained crystal fiber exceeds $100 \mathrm{~mm}$ with a diameter of about $1 \mathrm{~mm}$. The crystal fiber presents high transparency and there are no obvious inclusions and bubbles inside. 


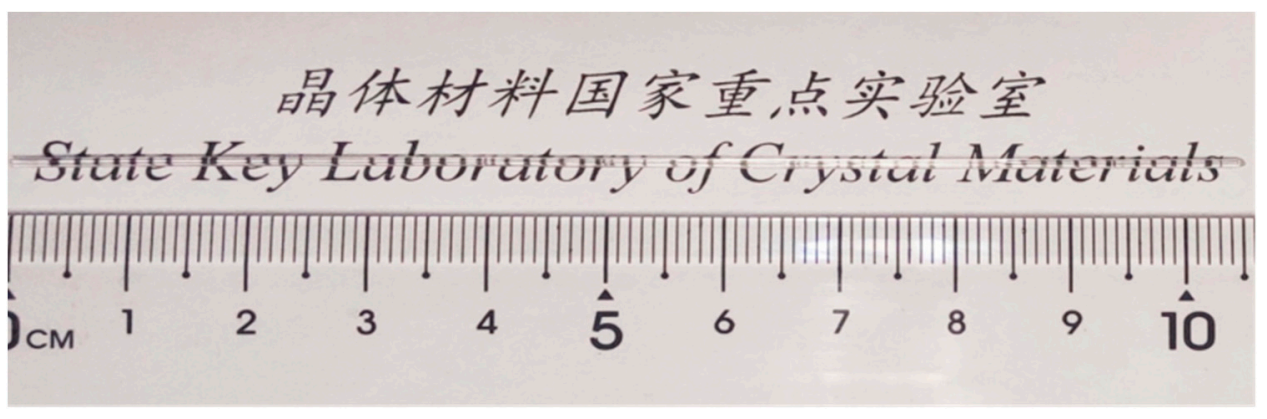

Figure 3. As-grown Yb:LuAG single-crystal fiber.

\subsection{Diameter Fluctuation}

The diameter fluctuation is one of the vital factors for evaluating the quality of SCFs, indicating the stability of the control system and the growth process. In order to obtain the diameter fluctuation of the Yb:LuAG SCF, one $1 \mathrm{~mm}$ diameter SCF was measured by a laser micrometer at every $1 \mathrm{~mm}$ along the fiber and, in total, 100 point data were collected.

\subsection{Laue Back-Reflection Measurements}

The growth direction and crystallinity of fibers were measured by a real-time Laue back diffractometer with a real-time back-reflection Laue camera system (Multiwire MWL 120 with Northstar software) along the axial and radial directions of the Yb:LuAG singlecrystal fibers.

\subsection{The Concentration and Distribution of $Y b^{3+}$}

The doping ion concentration of crystals was measured by an X-ray fluorescence spectrometer (Rigaku, zsx-primus II) (Rigaku, Matsubara-cho Akishima-shi, Tokyo, Japan). The test was carried out by pressing a crystal sample with a thickness of $2 \mathrm{~mm}$ into boric acid. In addition, the ion distribution of the end face and longitudinal direction was measured by a scanning electron microscope (G300 FE-SEM System) and electron probe microanalysis (EPMA-1720H, Shimadzu, Kyoto, Japan), respectively. The sample length was about $1 \mathrm{~mm}$, and the end face was polished for the mapping test. We selected an area of $150 \times 150 \mu \mathrm{m}^{2}$ on the end face randomly to perform the $\mathrm{Yb}^{3+}$ SEM mapping test and the EPMA line scan test of $\mathrm{Yb}^{3+}$ was carried out along the fiber axial direction.

\subsection{Spectroscopy}

The absorption spectrum was measured using a crystal wafer with two end faces polished. The spectrum data were recorded by an Agilent Cary 7000 UMS at room temperature in the range of 880-1050 $\mathrm{nm}$.

\subsection{Laser Experiments}

In order to study the laser performance of the prepared Yb:LuAG SCFs, a continuouswave laser experiment was designed. The laser experimental setup is shown in Figure 4. The pump source was a $940 \mathrm{~nm}$ semiconductor laser with a core diameter of $200 \mu \mathrm{m}$ and a numerical aperture (NA) of 0.22 . The magnification ratio of the focusing system was 1:1 and the resonant cavity length was $14 \mathrm{~cm}$. The plane input mirror (IM) was coated with high transmittance at the pump wavelength $(940-980 \mathrm{~nm})$ and high reflectivity from 1000 to $1100 \mathrm{~nm}$. The output mirror (OM) was partially reflective-coated from 1000 to $1100 \mathrm{~nm}$ with the transmittance of 5\%,10\% and 30\%. The crystal was wrapped in indium foil and placed in a copper radiator, and the water-cooling temperature was set to $17.5^{\circ} \mathrm{C}$ to reduce the thermal effect generated during the experiment. Yb:LuAG SCFs with the sizes of $\Phi 1 \mathrm{~mm} \times 8 \mathrm{~mm}$ and $\Phi 3 \mathrm{~mm} \times 8 \mathrm{~mm}$ were used in the experiment. 


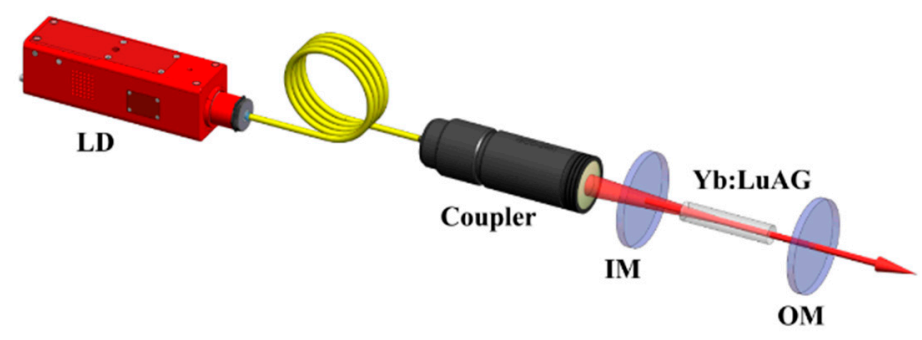

Figure 4. A schematic diagram of the Yb:LuAG single-crystal fiber (SCF) laser setup.

\section{Results and Discussion}

\subsection{Diameter Fluctuation}

As mentioned above, the uniformity of the fiber diameter greatly influences its application. The uniform diameter and the regular shape are conducive to achieving the total reflection of the pump light and reducing the light transmission loss. Compared with glass fibers, the greater viscosity of the SCF makes it more difficult to control the diameter. Improper parameters in the growth process such as the growth power and the vibration of the mechanical device will cause instability in the melting zone. Through the damping treatment of the mechanical devices and the optimization of the growth parameters, $\mathrm{Yb}$ : LuAG single-crystal fibers with a relatively uniform diameter were obtained. The diameter fluctuation of the LuAG single-crystal fiber is shown in Figure 5. The average fiber diameter is $988.3 \mu \mathrm{m}$, and the measured maximum and minimum diameters are 1015.6 and $978.6 \mu \mathrm{m}$, respectively. The diameter fluctuation of the Yb:LuAG SCF is 3.7\%, which is beneficial for conducting fiber laser experiments.

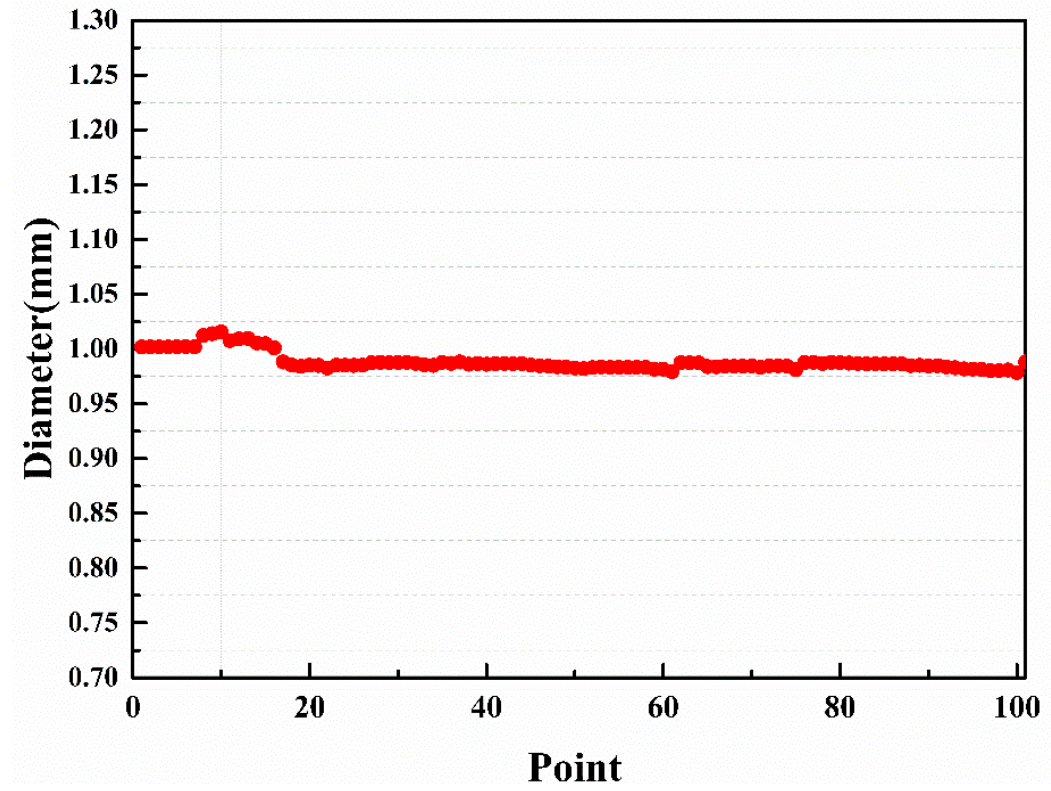

Figure 5. The diameter fluctuations of the Yb-doped LuAG SCF.

\subsection{Laue Back-Reflection Measurements}

Laue back-reflection measurement is usually used for crystal orientation and crystallinity tests. The X-ray beam is $0.5 \mathrm{~mm}$ in diameter, and the distance between the crystal and detector is $125 \mathrm{~mm}$. Figure $6 \mathrm{a}, \mathrm{b}$ show the test principles. It is demonstrated in Figure $6 a_{1}, a_{2}$ that the diffraction spots of the fiber end face are clear and the growth direction of the crystal is determined as $<111>$. The direction has a better optical quality than the other directions in cubic crystal systems. When the fiber moves along the axis to the test different areas, the diffraction spots are clear and consistent, as can be observed 
from Figure $6 b_{1}, b_{2}$. These results illustrate that the LuAG crystal fiber has good crystallinity and a consistent orientation.
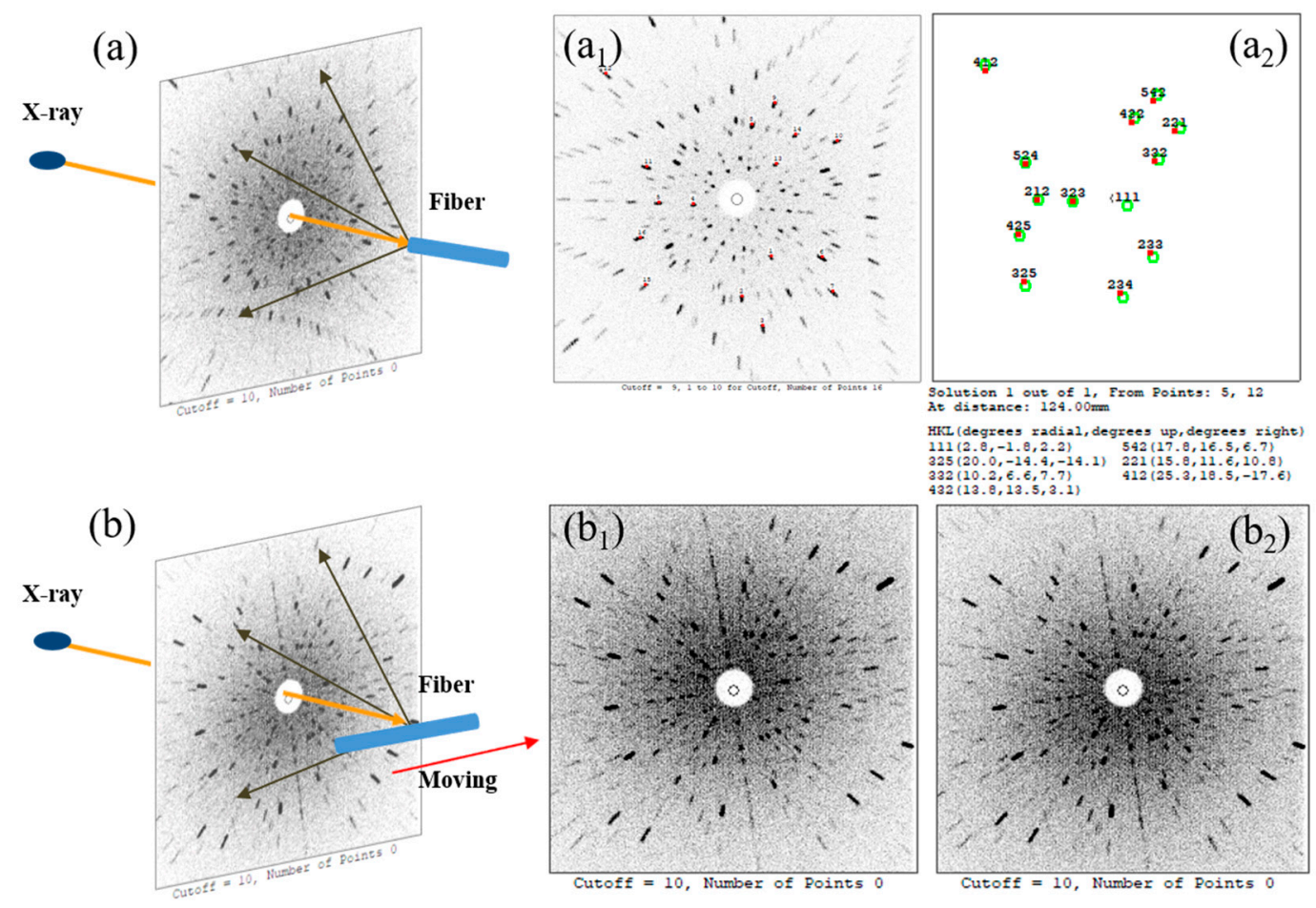

Figure 6. (a,b) Schematic diagrams of the Laue back-reflection measurements. $\left(\mathbf{a}_{1}, \mathbf{a}_{2}\right)$ Characteristic Laue back-reflection pattern and crystal orientation of fiber end face. $\left(\mathbf{b}_{1}, \mathbf{b}_{2}\right)$ Characteristic Laue back-reflection patterns at different positions with the X-ray beam hitting the SCF.

\subsection{The Concentration and Distribution of $Y b^{3+}$}

At the low doping concentration, the fluorescence lifetime of Yb:LuAG increases with the rise in the doping concentration, while the radiation capture and the fluorescence quenching effects occur at a high doping level [25]. The concentration of doping ions in this experiment was characterized by X-ray fluorescence (XRF), as shown in Table 1 . The concentration of $\mathrm{Yb}^{3+}$ in 1 and $3 \mathrm{~mm}$ diameter as-grown LuAG fiber samples is $7.26 \%$ and $10.71 \%$, respectively. The calculated segregation coefficient is close to 1 . Compared with other crystal growth methods, the segregation coefficient of $\mu$-PD technology is higher.

Table 1. The $\mathrm{Yb}^{3+}$ dopant concentration of the $\mathrm{Yb}:$ LuAG SCF.

\begin{tabular}{ccccc}
\hline Samples & Al (Mass\%) & Lu (Mass\%) & Yb (Mass\%) & Yb/Lu (at\%) \\
\hline$\Phi 1$ mm Yb:LuAG SCF & 10.6 & 83.4 & 5.99 & 7.26 \\
$\Phi 3$ mm Yb:LuAG SCF & 16.6 & 75.4 & 7.99 & 10.71 \\
\hline
\end{tabular}

In addition, the distribution of doped ions in the crystal fibers plays an important role in their laser performance. The aggregation of doped ions will bring in the non-uniform heat which is generated during the laser experiment process, consequently reducing the laser damage threshold of the crystal. The raw material melts evenly in the crucible when SCFs are grown by the $\mu$-PD method, which can further improve the uniformity of the composition. The $\mathrm{Yb}^{3+}$ ion distribution of the LuAG SCF was measured by EPMA and SEM, as shown in Figure 7. The results reflect that the $\mathrm{Yb}^{3+}$ is relatively evenly distributed in the LuAG SCF. 


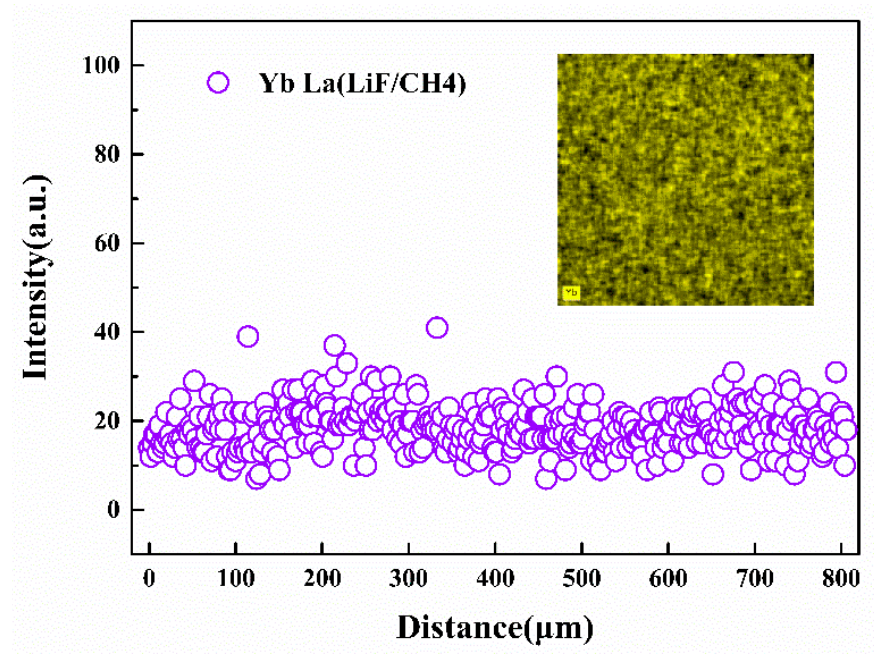

Figure 7. The axial and radial (inset) distributions of $\mathrm{Yb}^{3+}$ in the LuAG fiber.

\subsection{Spectral Property}

The absorption spectrum of the Yb:LuAG crystal between 880 and $1050 \mathrm{~nm}$ is shown in Figure 8. The main absorption peaks are located at 938 and 969 nm, respectively, corresponding to the ${ }^{2} \mathrm{~F}_{7 / 2} \rightarrow{ }^{2} \mathrm{~F}_{5 / 2}$ energy level transition of $\mathrm{Yb}^{3+}$. The absorption intensity at the wavelength of $938 \mathrm{~nm}$ is higher than that at $969 \mathrm{~nm}$ with the absorption coefficient of $9.87 \mathrm{~cm}^{-1}$, which provides a basis for the selection of the pump source. Further, a wider absorption peak is beneficial to improving the pumping efficiency, and the excitation of the pump wavelength of the matched laser diode (LD)pump source can be obtained without strict temperature control.

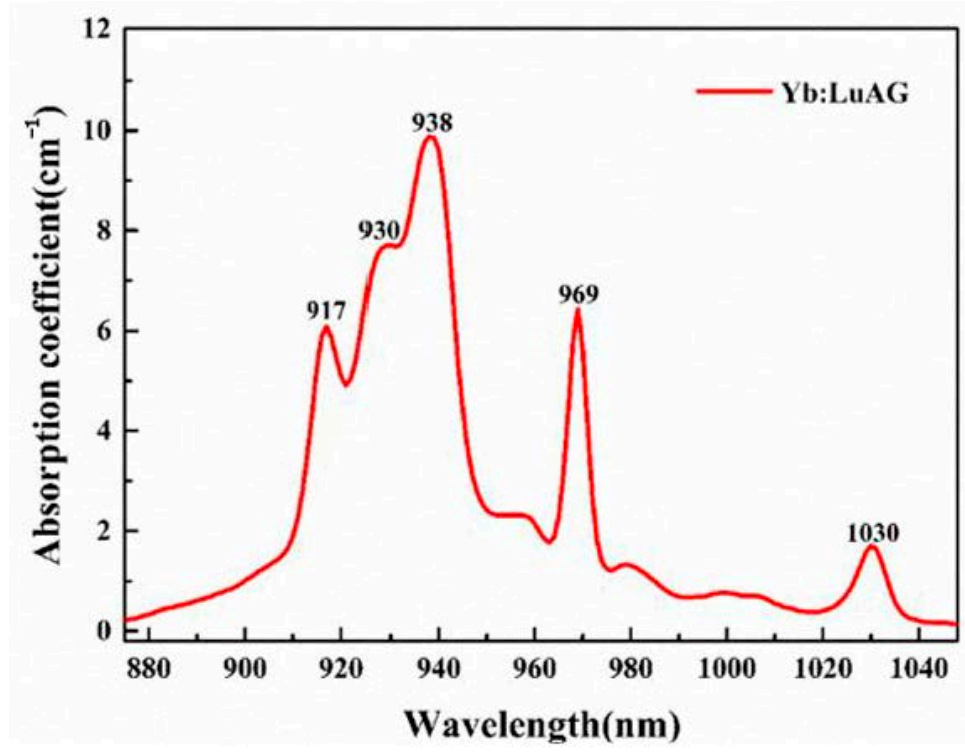

Figure 8. The absorption spectrum of the Yb:LuAG crystal in the wavelength range of 880-1050 nm.

\subsection{Laser Experiments}

Preliminary laser output verification of the as-grown Yb:LuAG SCFs was performed. With the output couplers of $5 \%$ and $30 \%$, the maximum continuous-wave $(\mathrm{CW})$ output laser power obtained by using the $1 \mathrm{~mm}$ diameter Yb:LuAG fiber is 1.53 and $1.96 \mathrm{~W}$, respectively, with the central wavelengths of 1032 and $1047 \mathrm{~nm}$ (Figure 9). Figure 10a shows the function of output laser power and absorption pump power. The slope efficiencies are $10.83 \%$ and $13.55 \%$, respectively, corresponding to an optical conversion efficiency of $6.99 \%$ and $10.09 \%$. The laser beam quality factor $\mathrm{M}^{2}$ at the highest output power is 1.08 with a good Gaussian 
distribution, indicating the excellent optical quality of the Yb:LuAG SCF (Figure 10b). A maximum output power of $4.7 \mathrm{~W}$ is achieved with a $3 \mathrm{~mm}$ diameter Yb:LuAG SCF at an absorbed pump power of $26.28 \mathrm{~W}$ (Figure 11). It is promising to achieve a higher laser output power through a higher pump power. In addition, further optimization of the laser system and coating of crystals also play an important role in achieving high-power and high-efficiency lasers.
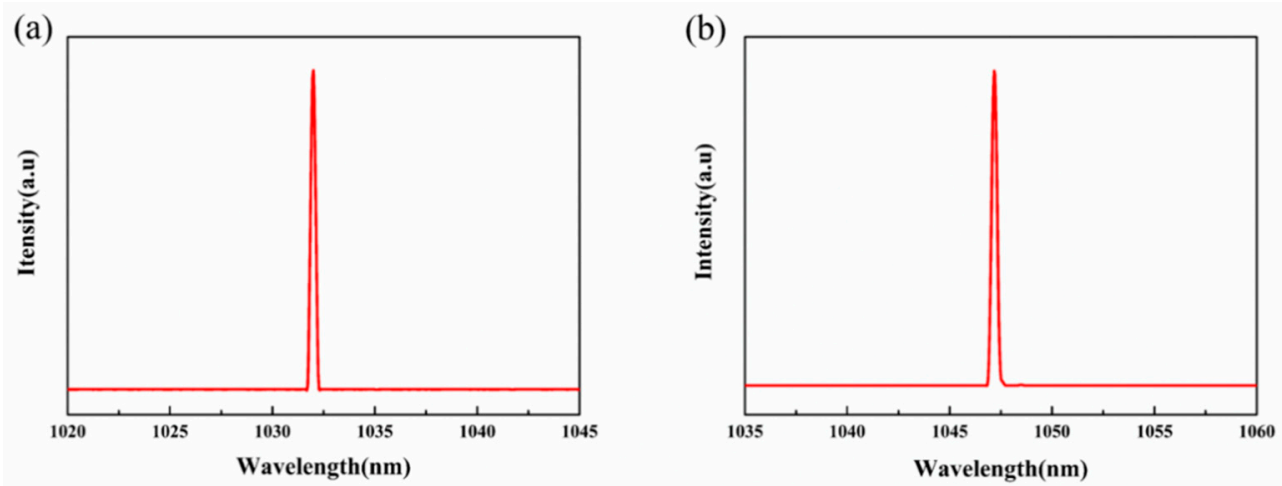

Figure 9. The laser spectra of the Yb:LuAG SCF with the central wavelengths of 1032 (a) and $1047 \mathrm{~nm}(\mathbf{b})$.

(a)

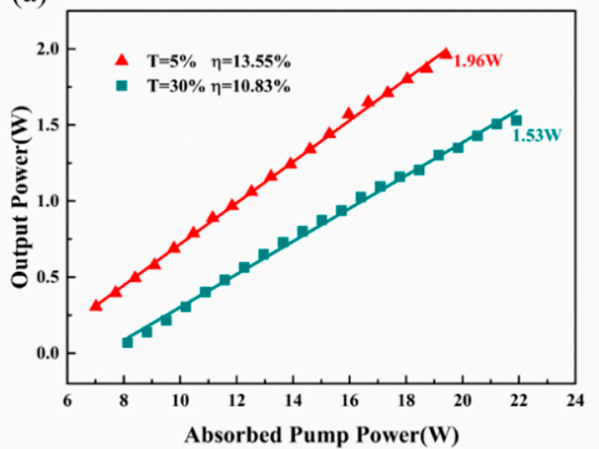

(b)

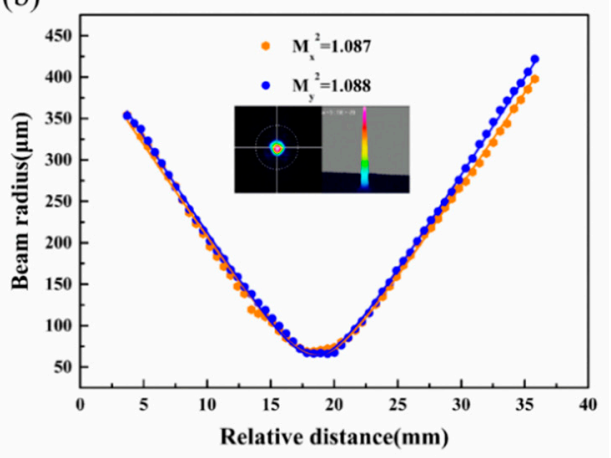

Figure 10. The continuous-wave (CW) laser performance (a) and the quality of the laser beam (b) at the maximum power output of the $1 \mathrm{~mm}$ diameter Yb:LuAG SCF.

(a)

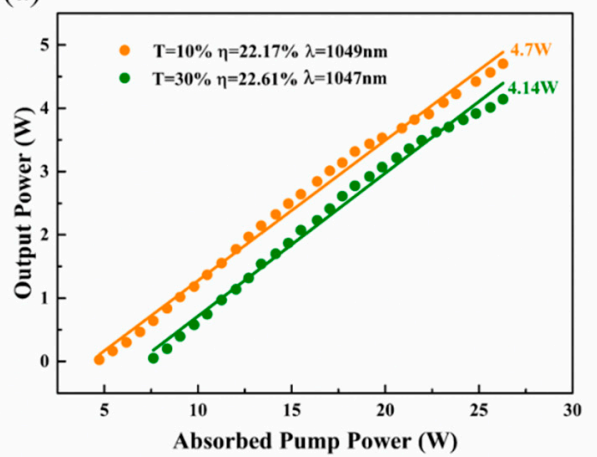

(b)

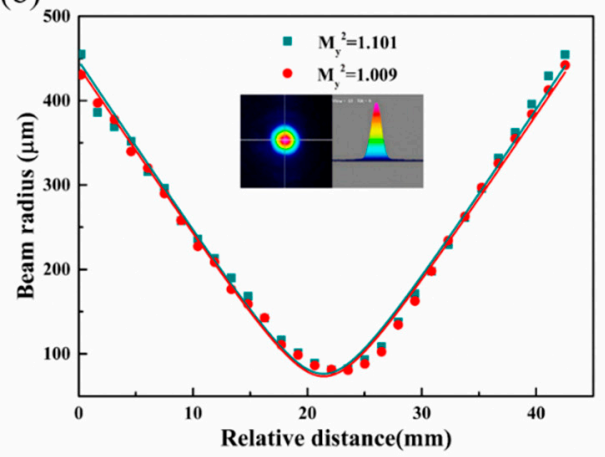

Figure 11. The CW laser performance (a) and the quality of the laser beam (b) at the maximum power output of the $3 \mathrm{~mm}$ diameter $\mathrm{Yb}:$ LuAG SCF.

\section{Conclusions}

In this paper, transparent and non-cracked $\mathrm{Yb}$ :LuAG single-crystal fibers were successfully grown through the $\mu$-PD technique. The diameter fluctuation of the crystal fiber is less than $5 \%$, suggesting that the growth conditions are well optimized and the mechanical 
control system is relatively stable. Hence, this $\mu$-PD technique shows a great prospect to be widely applied for $\mathrm{Yb}$ :LuAG SCF growth. The Laue back-scattering technology confirms that the fibers have good crystallinity. EPMA measurements prove that the $\mathrm{Yb}^{3+}$ ions are uniformly distributed in the LuAG fiber, which is crucial to the laser experiment. As a result, the maximum CW output power of the $1 \mathrm{~mm}$ Yb:LuAG SCF at 1032 and $1047 \mathrm{~nm}$ reaches 1.53 and $1.96 \mathrm{~W}$, respectively, with the beam factor $\mathrm{M}^{2}$ of 1.08 . In addition, a 4.7 W CW laser at $1049 \mathrm{~nm}$ is obtained by using a $3 \mathrm{~mm}$ diameter Yb:LuAG fiber with the slope efficiency of $22.17 \%$. All these results indicate that $\mathrm{Yb}$ :LuAG SCFs are a promising solid-state laser gain material for high-power laser applications.

Author Contributions: Conceptualization, A.W. and J.Z.; methodology, A.W. and B.W.; validation, A.W.; formal analysis, A.W. and S.W.; investigation, A.W., S.Y., X.M. and F.W.; data curation, A.W.; writing—original draft preparation, A.W.; writing—review and editing, A.W., T.W. and J.Z.; project administration, J.Z. and B.Z.; funding acquisition, J.Z. and Z.J. All authors have read and agreed to the published version of the manuscript.

Funding: This research was funded by the National Key Research and Development Program of China (2016YFB1102201), the Key Research and Development Program of Shandong Province (2018JMRH0207, 2018CXGC0410), the National Natural Science Foundation of China (51932004, 61975098, 61975095), the Natural Science Foundation of Shandong Province (ZR2018PEM007), the 111 Project 2.0 (Grant No: BP2018013) and the Open Funds of the State Key Laboratory of Rare Earth Resource Utilization (RERU2019010).

Conflicts of Interest: The authors declare no conflict of interest.

\section{References}

1. Extance, A. Military technology: Laser Weapons Get Real. Nat. News 2015, 521, 408-410. [CrossRef] [PubMed]

2. Brenier, A.; Wu, Y.; Fu, P.; Guo, R.; Jing, F. Evidence of self-frequency doubling from two inequivalent $\mathrm{Nd}^{3+}$ centers in the $\mathrm{La}_{2} \mathrm{CaB}_{10} \mathrm{O}_{19}: \mathrm{Nd}^{3+}$ bifunctional crystal. J. Appl. Phys. 2005, 98, 123528. [CrossRef]

3. Yu, H.H.; Zhang, H.; Wang, Z.; Wang, J.Y.; Yu, Y.G.; Jiang, M.H. Continuous-wave laser performance of $\mathrm{Nd}^{2} \mathrm{Lu}_{\mathrm{x}} \mathrm{Gd}_{1-\mathrm{x}} \mathrm{VO}_{4}$ operating at $1.34 \mu \mathrm{m}$. Laser Phys. Lett. 2008, 5, 181-184. [CrossRef]

4. Zuo, C.H.; Zhang, B.T.; He, J.L.; Dong, X.L.; Yang, K.J.; Huang, H.T.; Xu, J.L.; Zhao, S.; Dong, C.M.; Tao, X.T. The acousto-optical Q-switched Nd: GGG laser. Laser Phys. Lett. 2008, 5, 719-721. [CrossRef]

5. Nilsson, J.; Payne, D.N. High-Power Fiber Lasers. Science 2011, 332, 921-922. [CrossRef] [PubMed]

6. Kratky, A.; Schuöcker, D.; Liedl, G. Processing with kW fibre lasers: Advantages and limits. In Proceedings of the XVII International Symposium on Gas Flow and Chemical Lasers and High Power Lasers, Lisbon, Portugal, 15-19 September 2008; SPIE: Bellingham, WA, USA, 2008; Volume 7131, p. 71311X.

7. Parthasarathy, T.A.; Hay, R.S.; Fair, G.; Hopkins, F.K. Predicted performance limits of yttrium aluminum garnet fiber lasers. Opt. Eng. 2010, 49, 094302. [CrossRef]

8. Délen, X.; Piehler, S.; Didierjean, J.; Aubry, N.; Voss, A.; Ahmed, M.A.; Graf, T.; Balembois, F.; Georges, P. 250 W single-crystal fiber Yb:YAG laser. Opt. Lett. 2012, 37, 2898-2900. [CrossRef]

9. Kalaycioglu, H.; Sennaroglu, A.; Kurt, A.; Özen, G. Spectroscopic analysis of $\mathrm{Tm}^{3+}$ :LuAG. J. Phys. Condens. Matter 2007, 19, 036208. [CrossRef]

10. Petrosyan, A.G.; Shirinyan, G.O. Peculiarities of crystallization of rare-earth garnets from non-stoichiometric melts. Inorg. Mater. 1993, 29, 258-261.

11. Beil, K.; Fredrich-Thornton, S.T.; Tellkamp, F.; Peters, R.; Huber, G. Thermal and laser properties of Yb:LuAG for kW thin disk lasers. Opt. Express 2010, 18, 20712-20722. [CrossRef]

12. Veselský, K.; Šulc, J.; Jelínková, H.; Nejezchleb, K.; Škoda, V. Yb Doping Concentration and Temperature Influence on Yb:LuAG Thermal Lensing. In Proceedings of the SPIE LASE: Solid State Lasers XXV: Technology and Devices, San Francisco, CA, USA, 15-18 February 2016; SPIE: Bellingham, WA, USA, 2016.

13. Brenier, A.; Guyot, Y.; Cañibano, H.; Boulon, G.; Ródenas, A.; Jaque, D.; Eganyan, A.; Petrosyan, A.G. Growth, spectroscopic, and laser properties of $\mathrm{Yb}^{3+}$-doped $\mathrm{Lu}_{3} \mathrm{Al}_{5} \mathrm{O}_{12}$ garnet crystal. J. Opt. Soc. Am. B 2006, 23, 676-683. [CrossRef]

14. Dong, J.; Ueda, K.I.; Kaminskii, A.A. Efficient passively Q-switched Yb: LuAG microchip laser. Opt. Lett. 2007, 32, 3266-3268. [CrossRef] [PubMed]

15. Euler, F.; Bruce, J.A. Oxygen coordinates of compounds with garnet structure. Acta Crystallogr. 1965, 19, 971-978. [CrossRef]

16. Nakao, H.; Shirakawa, A.; Ueda, K.; Yagi, H.; Yanagitani, T.; Weichelt, B.; Wentsch, K.; Ahmed, M.A.; Graf, T. Yb ${ }^{3+}$-Doped $\mathrm{Lu}_{3} \mathrm{Al}_{5} \mathrm{O}_{12}$ Ceramic Thin-Disk Laser. In Proceedings of the Conference on Lasers and Electro-Optics Pacific Rim, Kyoto, Japan, 30 June-4 July 2013.

17. Klemens, P.G. Thermal Resistance due to Point Defects at High Temperatures. Phys. Rev. 1960, 119, 507-509. [CrossRef] 
18. $\mathrm{Mu}, \mathrm{X}$; Meissner, S.K.; E Meissner, H. Laser diode pumped high efficiency Yb:YAG crystalline fiber waveguide lasers. In Proceedings of the SPIE LASE: Solid State Lasers XXIV: Technology and Devices, San Francisco, CA, USA, 8-10 February 2015; SPIE: Bellingham, WA, USA, 2015; Volume 9342, p. 934209.

19. Johnson, L.F.; Geusic, J.E.; Van Uiter, L.G. Coherent oscillations from $\mathrm{Tm}^{3+}, \mathrm{Ho}^{3+}, \mathrm{Yb}^{3+}$ and $\mathrm{Er}^{3+}$ ions in yttrium aluminum garnet. Appl. Phys. Lett. 1965, 7, 127-129. [CrossRef]

20. Sangla, D.; Aubry, N.; Nehari, A.; Brenier, A.; Tillement, O.; Lebbou, K.; Balembois, F.; Georges, P.; Perrodin, D.; Didierjean, J. $\mathrm{Yb}$-doped $\mathrm{Lu}_{3} \mathrm{Al}_{5} \mathrm{O}_{12}$ bers single crystals grown under stationary stable state for laser application. J. Cryst. Growth 2009, 312, 125-130. [CrossRef]

21. Veronesi, S.; Zhang, Y.; Tonelli, M.; Agnesi, A.; Greborio, A.; Pirzio, F.; Reali, G. Spectroscopy and efficient laser emission of Yb ${ }^{3+}$ : LuAG single crystal grown by $\mu$-PD. Opt. Commun. 2012, 285, 315-321. [CrossRef]

22. Wang, T.; Zhang, J.; Zhang, N.; Wang, S.; Wu, B.; Jia, Z.; Tao, X. The characteristics of high-quality Yb:YAG single crystal fibers grown by a LHPG method and the effects of their discoloration. RSC Adv. 2019, 9, 22567-22575. [CrossRef]

23. Che, Z.; Zhang, J.; Wu, B.; Hu, Q.; Jia, Z. Investigation of $\mathrm{Y}_{2.1} \mathrm{Er}_{0.9}\left(\mathrm{Sc}_{\mathrm{x}} \mathrm{Ga}_{1-\mathrm{x}}\right)_{5} \mathrm{O}_{12}$ Matrix Components on the Spectral Prop-erties around $3.0 \mu \mathrm{m}$ by Micro-Pulling-Down Method. Crystals 2019, 9, 138. [CrossRef]

24. Wu, B.; Nie, H.; Wang, A.; Zhang, J.; Jia, Z.; Zhang, B.; Fu, X.; Hu, Q.; He, J.; Tao, X. Factors influencing optical uniformity of YAG single-crystal fiber grown by micro-pulling-down technology. Cryst. Eng. Comm. 2019, 21, 6929-6934. [CrossRef]

25. Toncelli, A.; Alshourbagy, M.; Tonelli, M. Optical properties of $\mathrm{Yb}^{3+}$ doped $\mathrm{Lu}_{3} \mathrm{Al}_{5} \mathrm{O}_{12}$ crystal fibers grown by $\mu$-pulling down technique. J. App. Phys. 2008, 104, 350-676. [CrossRef] 